\title{
Theory and ontology in behavioural science
}

To the Editor - In a recent Perspective ${ }^{1}$, Muthukrishna and Henrich ( $\mathrm{MH}$ ) argue that an important and overlooked driver for the replication crisis in the social and behavioural sciences is "the lack of a cumulative theoretical framework." We have previously written about the importance of theory for human behaviour research ${ }^{2,3}$, and we agree that harnessing theories helps to enable cumulative science, by coordinating evidence and synthesis, providing a rationale for predictions and giving a basis for interpreting new findings ${ }^{4}$. The more diverse the nature of the empirical verification that supports the same theoretical conclusion, the more confident we can be that it is true $^{5,6}$. Even so, it will be difficult for researchers across the behavioural sciences to agree on any one theory, as theories vary in their perspective and scope. The ensuing challenge, as we see it, is to identify a framework that can integrate findings arising from different theoretical approaches, to develop as comprehensive a view as possible about what is known. This depends on systematically linking evidence to theory in a way that allows determination of which theoretical propositions are more or less supported by the available evidence, across the multiple domains, fields or disciplines from which evidence may arise ${ }^{6}$.

Theories have a specific scope and subject matter and describe entities and relationships. They can be formally represented in ontologies (Fig. 1): structured, computational representations of entities and relationships in a given domain ${ }^{7,8}$.

As computable representations of knowledge and part of the 'data science' family of semantic technologies, ontologies serve as hubs around which evidence can be aggregated and theoretical debates can be resolved. Explicitly defining entities from across different theories enables those theories to be connected. For example, an entity such as 'perceived

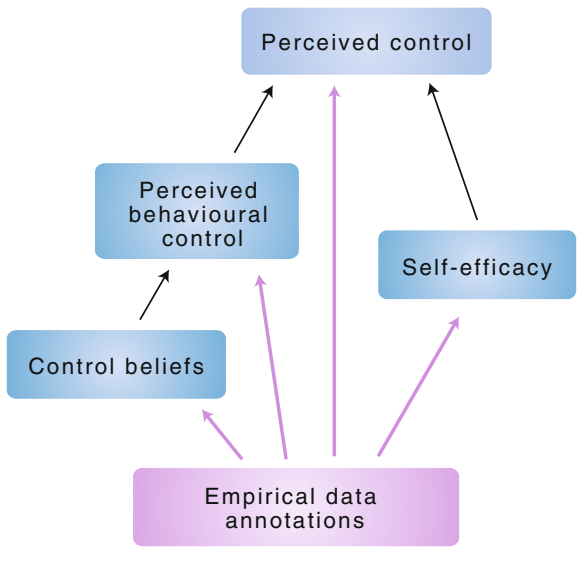

Fig. 1 | Illustration of an ontology representing entities from different theories.

control' might encompass the entities 'perceived behavioural control' (from the Theory of Planned Behaviour), 'selfefficacy' (from Social Cognitive Theory) and 'control representation' (from the Common Sense Model) ${ }^{9}$. Two theories are only comparable-and may therefore be congruent or contradictory-to the extent that they are about the same entities. Theoretical integration entails identifying the entities of the different theories to determine when different theories are addressing the same, overlapping, distinct, or poorly specified entities. A project that aims to achieve this objective has already been initiated ${ }^{3}$.

$\mathrm{MH}$ argue for the benefit of "unifying overarching theories," drawing on examples from the natural sciences: special relativity, the periodic table and Darwin's evolutionary theory. Each of these has its own very specific domain and scope, and their successes are due in part to the clear definition of entities and the relations between those entities, allowing integration with other theories addressing the same entities. In the behavioural sciences, one key challenge in adopting any theory is the plethora of competing alternative entities and the field's lack of a principled approach to integrate across or select between them for use in interpreting a given phenomenon. We propose an integrative approach based on ontologies ${ }^{10}$. This requires theory authors to become more explicit about the tenets of their theories and to define the entities and relations therein. Ontology annotation of empirical findings to an integrated knowledge base of theoretical entities then provides a direct connection from entities to evidence, regardless of the theoretical background that led to the generation of the evidence, providing a firm grounding for a cumulative science.

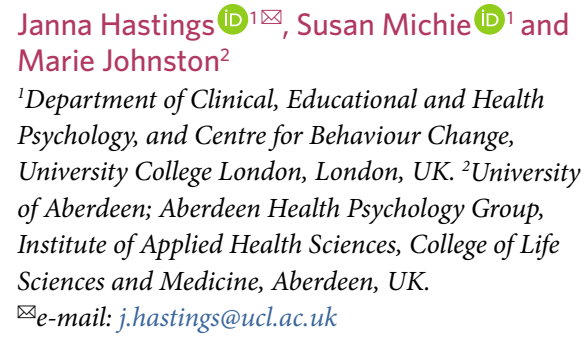

Published online: 17 February 2020 https://doi.org/10.1038/s41562-020-0826-9

References

1. Muthukrishna, M. \& Henrich, J. Nat. Hum. Behav. 3, 221-229 (2019).

2. Davis, R., Campbell, R., Hildon, Z., Hobbs, L. \& Michie, S. Health Psychol. Rev. 9, 323-344 (2015).

3. West, R. et al. Nat. Hum. Behav. 3, 526-536 (2019).

4. Freedland, K. E. Health Psychol. 38, 277-289 (2019).

5. Munafò, M. R. \& Davey Smith, G. Nature 553, 399-401 (2018).

6. Munafò, M. R. et al. Nat. Hum. Behav. 1, 21 (2017).

7. Michie, S. et al. Implement. Sci. 12, 121 (2017).

8. Hastings, J. Methods Mol. Biol. 1446, 3-13 (2017).

9. Michie, S., West, R., Campbell, R., Brown, J. \& Gainforth, H. An $A B C$ of Behaviour Change Theories (Silverback Publishing, 2014)

10. Hastings, J., Michie, S. \& Johnston, M. (2019) Theory and ontology in building cumulative behavioural science. Preprint available at $O S F$ https://osf.io/9te3x/ (2019).

Competing interests

The authors declare no competing interests. 